\title{
Trafficking Macrophage Migration Using Reporter Gene Imaging with Human Sodium Iodide Symporter in Animal Models of Inflammation
}

Ji Hyoung Seo ${ }^{* 1,2}$, Yong Hyun Jeon*1, Yong Jin Lee ${ }^{1}$, Gil Sook Yoon ${ }^{3}$, Dong-Il Won ${ }^{4}$, Jeoung-Hee Ha ${ }^{5}$, Shin Young Jeong ${ }^{1}$, Sang Woo Lee ${ }^{1}$, Byeong Cheol Ahn ${ }^{1}$, and Jaetae Lee ${ }^{1}$

${ }^{1}$ Department of Nuclear Medicine, Kyungpook National University, Daegu, Korea; ${ }^{2}$ Department of Nuclear Medicine, Haeundae Paik Hospital, Inje University College of Medicine, Busan, Korea; ${ }^{3}$ Department of Pathology, Kyungpook National University, Daegu, Korea; ${ }^{4}$ Department of Clinical Pathology, Kyungpook National University, Daegu, Korea; and ${ }^{5}$ Department of Pharmacology, Kyungpook National University, Daegu, Korea

The aim of this study was to investigate the feasibility of nuclear molecular imaging using the human sodium iodide symporter (hNIS) as a reporter gene to monitor macrophage migration toward the inflammatory foci. Methods: A stable macrophage cell line coexpressing hNIS and green fluorescent protein (GFP) genes (RAW264.7/hNIS-GFP and $\mathrm{R}_{\text {NIS }}$ cell) was established from an immortalized macrophage cell line (RAW264.7 cells). ${ }^{125} \mathrm{I}$ uptake was determined (for hNIS protein functional activity), and flow cytometry analysis (to examine GFP gene expression), a cell proliferation assay, a cytokine assay, and a phagocytic activity assay were performed. 99mTc-pertechnetate images were acquired at $1 \mathrm{~d}$ after subcutaneous inoculation of $\mathrm{R}_{\mathrm{NIS}}$ cells in nude mice. Chemical inflammation was induced for in vivo imaging in the thigh of nude mice by turpentine oil injection. Small-animal PET with ${ }^{18} \mathrm{~F}-\mathrm{FDG}$ and ${ }^{124} \mathrm{I}$ was performed with an intravenous administration of RAW264.7 or $\mathrm{R}_{\mathrm{NIS}}$ cells in inflammation-induced animals. Results: The expression of hNIS and GFP genes was confirmed in $\mathrm{R}_{\mathrm{NIS}}$ cells by flow cytometry and immunofluorescent staining. ${ }^{125} \mathrm{I}$ uptake was about 67 times higher in $\mathrm{R}_{\text {NIS }}$ cells than in RAW264.7 cells. No significant difference was observed in cell proliferation, cytokine production, and phagocytic activity between RAW264.7 and $R_{\text {NIS }}$ cells. 99mTc-pertechnetate imaging revealed increased tracer uptake at the inoculation site. PET with ${ }^{124}$ I demonstrated a donut-shaped uptake, correlating with uptake shown by the ${ }^{18}$ F-FDG PET images, at the inflammation site of mice administered $\mathrm{R}_{\mathrm{NIS}}$ cells. ${ }^{124} \mathrm{I}$ uptake (percentage injected dose per gram) was about 2.12 times higher at the inflammation site in the $\mathrm{R}_{\mathrm{NIS}}$ mice than in RAW264.7 mice. By immunohistochemistry, the migration of macrophages was further confirmed by positive staining for GFP and hNIS at the inflammation site of $\mathrm{R}_{\mathrm{NIS}}$ mice. Conclusion: These data support the feasibility of hNIS reporter gene imaging to monitor the macrophage migration toward an inflammatory lesion. Macrophages expressing hNIS may provide a new strategy to investigate the cellular behavior seen with inflammatory response in a preclinical model.

Received Apr. 7, 2010; revision accepted Jul. 14, 2010.

For correspondence contact: Jaetae Lee, Department of Nuclear Medicine, Kyungpook National University Hospital, 50 Samduk 2-ga,

Daegu, 700-721, Republic of Korea.

E-mail: jaetae@knu.ac.kr

${ }^{*}$ Contributed equally to this work.

COPYRIGHT @ 2010 by the Society of Nuclear Medicine, Inc.
Key Words: reporter gene imaging; sodium iodide symporter; macrophage; ${ }^{124}$ I PET

J Nucl Med 2010; 51:1637-1643

DOI: 10.2967/jnumed.110.077891

M acrophages are important immune cells implicated in onset, progression, and manifestation of various pathologic processes, such as growth and spread of malignant tumors, and chronic inflammatory diseases (1). Macrophages act as hosts and reservoirs for pathogens and produce proinflammatory or proangiogenic mediators (2-4). Various attempts have been made to visualize the macrophage-mediated inflammatory process in vivo, but specific visualization of these cells in the inflammatory process remains a considerable task with the currently available imaging modalities, such as radionuclide imaging or MRI, which are considered appropriate to visualize the inflammatory changes in tissue (5). Nuclear methods such as PET and SPECT exhibit relatively poor spatial resolution, but their good sensitivity is considered a powerful feature in molecular imaging (6). Development of molecular imaging with PET has been made much easier as assays in cell culture and small-animal models are first validated and then the same tracer used in those procedures is used in established clinical PET sites (7). Therefore PET-based molecular-genetic imaging has developed rapidly in the past decade.

Most current molecular-genetic imaging strategies are based on a reporter gene and a complementary reporter probe. The reporter gene is constitutively expressed, and reporter imaging is used to track gene therapy vectors or transduced cells in the body (8). One such example of a reporter gene system is the sodium iodide symporter (NIS). NIS reporter gene imaging has the advantages of using known radiotracers such as ${ }^{99 \mathrm{~m}} \mathrm{Tc}$-pertechnetate and radioiodine. Additional benefits are that NIS is nonimmunogenic, with no observed adverse effects on cell viability and function (9), and this technique does not require a complex probe synthesis. 
The aim of this study was to investigate the feasibility of nuclear molecular imaging using the human sodium iodide symporter (hNIS) as a reporter gene that functions to monitor macrophage migration toward a chemically induced inflammatory lesion. An immortalized macrophage cell line coexpressing hNIS and green fluorescent protein (GFP) genes (RAW264.7/hNIS-GFP and $\mathrm{R}_{\mathrm{NIS}}$ cells) was established. ${ }^{125}$ I uptake was determined, and flow cytometry analysis, a cell proliferation assay, a cytokine assay, and a phagocytic activity assay were performed. Small-animal PET with ${ }^{18} \mathrm{~F}$-FDG and ${ }^{124} \mathrm{I}$ was performed with an intravenous administration of RAW264.7 or $R_{\text {NIS }}$ cells in inflammation-induced animals.

\section{MATERIALS AND METHODS}

\section{Cells and Animals}

Specific pathogen-free 6-wk-old female BALB/c nude mice $(n=20)$ were obtained from SLC Inc. All animal experiment protocols were approved by the Committee for the Handling and Use of Animals, Kyungpook National University. The murine macrophage RAW264.7 cell line was kindly provided by Dr. Chul Woo Kim (Seoul National University).

\section{Establishment of Stable Macrophage Cell Line Coexpressing hNIS and GFP Genes}

To produce the lentivirus coexpressing hNIS and GFP genes, human embryonic kidney 293 cells were plated at $5 \times 10^{6}$ cells in a $100-\mathrm{cm}$ tissue culture plate, and cells were cotransfected with replication-incompetent lentiviral vector (pLenti/PGK-hNISIRES-GFP), vesicular stomatitis virus glycoprotein, and dR8.74 using a calcium phosphate transfection kit and reagents as described by the manufacturer (Invitrogen). Growth medium was changed at $24 \mathrm{~h}$, and lentivirus-containing supernatant was harvested at $48 \mathrm{~h}$. Harvested supernatant was centrifuged at $4^{\circ} \mathrm{C}$, $3,000 \mathrm{rpm}$, for $15 \mathrm{~min}$ and stored at $-80^{\circ} \mathrm{C}$.

To establish a stable macrophage cell line coexpressing hNIS and GFP, RAW264.7 cells were transduced by adding $1 \mathrm{~mL}$ of lentivirus-containing supernatant and $10 \mu \mathrm{g}$ of polybrene per milliliter. After 48 h, GFP-positive cells were enriched using FACSaria (BD Biosciences). The selection procedure was performed twice for $6 \mathrm{wk}$.

\section{Immunofluorescent Staining}

RAW264.7 or $\mathrm{R}_{\mathrm{NIS}}$ cells were seeded at a density of $4 \times 10^{4}$ cells per well on collagen-precoated chamber slides (Nunc) and grown for $24 \mathrm{~h}$. The hNIS-specific immunostaining was performed with a Cytofix/cytoperm kit (BD Pharmingen). The cells were washed twice with phosphate-buffered saline (PBS). The washed cells were fixed and permeabilized with buffer (eBioscience) for $20 \mathrm{~min}$ at $4^{\circ} \mathrm{C}$. After that, the cells were washed with $1 \mathrm{X}$ BD Perm/Wash buffer (BD Bioscience) and stained with anti-hNIS antibody (Milipore) diluted with 1X BD Perm/Wash buffer for 1 $\mathrm{h}$ at room temperature. After the cells were washed 3 times with 1X BD Perm/Wash buffer, Alexa Fluor 568-conjugated secondary antibody (Molecular Probes, Inc.) was applied for $40 \mathrm{~min}$ at room temperature. The cells were washed 3 times with 1X BD Perm/ Wash buffer. The slides were mounted with Vectashield mounting medium (Vector Laboratories), covered with glass cover slips, and examined using a laser confocal scanning system (TCS SP2; Leica).

\section{Radioiodide Uptake Assays}

Parental RAW264.7 cells and $\mathrm{R}_{\mathrm{NIS}}$ cells $\left(5 \times 10^{4}, 1 \times 10^{5}, 3 \times\right.$ $10^{5}$, and $5 \times 10^{5}$ ) were plated in 24-well plates and cultured with Dulbecco's modified Eagle's medium containing $10 \%$ fetal bovine serum for $24 \mathrm{~h} .{ }^{125} \mathrm{I}$ uptake was determined by incubating the cells with $500 \mu \mathrm{L}$ of Hank's balanced salt solution (bHBSS) (GibcoBRL Co.) containing $3.7 \mathrm{kBq}(0.1 \mu \mathrm{Ci})$ of carrier-free ${ }^{125} \mathrm{I}-\mathrm{NaI}$ and $10 \mu \mathrm{M} \mathrm{NaI}$ at $37^{\circ} \mathrm{C}$ for $30 \mathrm{~min}$. After incubation, the cells were quickly washed twice with bHBSS and detached using $500 \mu \mathrm{L}$ of trypsin. Radioactivity was measured using a $\gamma$-counter.

Iodide uptake was measured at various times points. Cells $(200,000)$ were plated and incubated with $500 \mu \mathrm{L}$ of bHBSS containing $3.7 \mathrm{kBq}(0.1 \mu \mathrm{Ci})$ of carrier-free ${ }^{125} \mathrm{I}-\mathrm{NaI}$ and $10 \mu \mathrm{M}$ $\mathrm{NaI}$ at different times $(1,5,15,30,45$, and $60 \mathrm{~min})$. For the blocking study, cells were incubated in ${ }^{125} \mathrm{I}-\mathrm{NaI}$ medium either with or without $100 \mu \mathrm{M}$ of potassium perchlorate.

For the iodide efflux study, cells were incubated with $10 \mu \mathrm{M}$ $\mathrm{NaI}$ and $3.7 \mathrm{kBq}(0.1 \mu \mathrm{Ci})$ of carrier-free ${ }^{125} \mathrm{I}-\mathrm{NaI}$ in $500 \mu \mathrm{L}$ of bHBSS incubation buffer at $37^{\circ} \mathrm{C}$ for $30 \mathrm{~min}$. Cells were then washed twice and incubated with bHBSS at $37^{\circ} \mathrm{C}$. bHBSS was removed at different times $(0,1,3,6,9,12,15,18,21,24,27$, and $30 \mathrm{~min}$ ), and its radioactivity was measured. Cells were collected after the last time, and residual radioactivity was determined as counts per minute. All data are expressed as the percentage remaining for the total activity.

\section{Cell Proliferation Assay}

Parental RAW264.7 cells and $\mathrm{R}_{\mathrm{NIS}}$ cell cells were plated at $2 \times$ $10^{4}$ per well in $96-$ well plates. Two days later, a $10-\mu \mathrm{L}$ cell count solution (CCK-8; Dojindo) was added to the culture medium of cells, and plates were incubated at $37^{\circ} \mathrm{C}$ for $4 \mathrm{~h}$. Absorbance was measured at a wavelength of $450 \mathrm{~nm}$ using a microplate reader (Bio-Rad Laboratories).

\section{Cytokine Assay}

To assess the functionality of macrophages, cytokine production was measured. Parental RAW264.7 and $\mathrm{R}_{\mathrm{NIS}}$ cells were stimulated with $1 \mu \mathrm{g}$ of lipopolysaccharide per milliliter. After $24 \mathrm{~h}$, supernatants were harvested and analyzed for IL-12p70 and tumor necrosis factor (TNF)- $\alpha$ by enzyme-linked immunosorbent assay using commercially available kits (BD Pharmingen). Cytokine concentrations were quantified using a microplate reader.

\section{Phagocytic Activity Assay}

To determine the effects of virus infection on phagocytosis of macrophage cells, we determined phagocytic activity of RAW264.7 and $\mathrm{R}_{\mathrm{NIS}}$ cells using 7-aminoactinomycin D (7AAD; BD Biosciences Pharmingen)-stained Escherichia coli and fluorescent-activated cell sorter (FACS) analysis (10-13). E. coli $\left(1 \times 10^{8}\right)$ in $1 \mathrm{~mL}$ of PBS was incubated at $60^{\circ} \mathrm{C}$ for $1 \mathrm{~h}$ for sterilization, and $1 \mathrm{~mL}$ of sterilized $E$. coli was further coincubated with $50 \mu \mathrm{L}$ of 7 -AAD for $2 \mathrm{~h}$ at room temperature. For opsonization of $E$. coli to allow phagocytosis by macrophages, $5 \mu \mathrm{L}$ of 7-AAD-stainined E. coli were incubated with $1 \mu \mathrm{L}$ of human serum for $20 \mathrm{~min}$ at room temperature. Opsonized $E$. coli stained with 7-AAD was cocultured with $2.5 \times 10^{5}$ RAW264.7 and $\mathrm{R}_{\mathrm{NIS}}$ cells, which were incubated in $50 \mu \mathrm{L}$ of RPMI 1640 culture medium (Hyclone) containing 10\% fetal bovine serum for $1 \mathrm{~h}$ at either $4^{\circ} \mathrm{C}$ or $37^{\circ} \mathrm{C}$. At $1 \mathrm{~h}$ after incubation, the samples were washed with cold PBS twice, and fluorescence activities were analyzed using a FACS Calibur (BD Bioscience). The phagocytic 
activity of each cell was expressed as increases in the percentage of 7-AAD-positive RAW264.7 or $\mathrm{R}_{\mathrm{NIS}}$ cells, compared with those treated with nonstained $E$. coli.

\section{Nuclear Imaging with $99 \mathrm{~m}$ Tc-Pertechnetate}

$\mathrm{R}_{\mathrm{NIS}}$ cells, $8 \times 10^{6}$ and $2 \times 10^{7}$, were subcutaneously implanted in a volume of $0.1 \mathrm{~mL}$ into the right and left thighs, respectively, of specific pathogen-free BALB/c nude mice (6-wk-old female, $n=3)$. Twenty-four hours after inoculation, $18.5 \mathrm{MBq}(500 \mu \mathrm{Ci})$ of ${ }^{99 \mathrm{~m}} \mathrm{Tc}$-pertechnetate in $100 \mu \mathrm{L}$ of PBS was injected intravenously, and a whole-body scan was obtained at $30 \mathrm{~min}$ after injection using a $\gamma$-camera equipped with a pinhole collimator (Infinia II; GE Healthcare).

\section{Small-Animal PET with ${ }^{18}$ F-FDG and ${ }^{124} \|$ in Inflammation Animal Model}

The animal experimental procedure is demonstrated schematically in Supplemental Figure 1 (supplemental materials are available online only at http://jnm.snmjournals.org). Turpentine oil $(50 \mu \mathrm{L} /$ mouse) was intramuscularly injected into the right thigh of nude mice ( 5 mice/group). Lipopolysaccharide $(1 \mu \mathrm{g} /$ $\mathrm{mL}$ ) was injected to promote the inflammatory process. Inflammation was confirmed $7 \mathrm{~d}$ later, and parental RAW264.7 cells or $\mathrm{R}_{\mathrm{NIS}}$ cells $\left(3 \times 10^{6} /\right.$ mouse $)$ were intravenously injected into inflammation-induced mice. Seven days after macrophage injection, $3.7 \mathrm{MBq}(100 \mu \mathrm{Ci})$ of ${ }^{124} \mathrm{I}$ was administered into mice. After $1 \mathrm{~h}$, mice were placed prone on the bed of the smallanimal PET scanner. ${ }^{18} \mathrm{~F}$-FDG PET was performed after intravenous injection of $11.1 \mathrm{MBq}(300 \mu \mathrm{Ci})$ of ${ }^{18} \mathrm{~F}-\mathrm{FDG}$ in mice under general anesthesia with $1 \%-2 \%$ isoflurane in $100 \% \mathrm{O}_{2}$ during injection and imaging. The images were recon- structed with a 2-dimensional ordered-subsets expectation maximization algorithm. Corrections were not done for attenuation or scattering. Accumulation of ${ }^{124} \mathrm{I}$ in the inflamed tissue was quantitatively determined from small-animal PET images by placing a region of interest on the inflamed tissue visualized in coronal ${ }^{124} \mathrm{I}$ images and expressed as percentage injected dose per gram.

\section{Histopathologic Analysis}

Tissues that showed inflammation were removed from mice and preserved in $10 \%$ formalin until required. All inflammatory tissues were embedded in paraffin and sectioned at $5 \mu \mathrm{m}$. For immunohistologic analysis of hNIS and GFP expression, the paraffin sections were incubated for $1 \mathrm{~h}$ with anti-hNIS or anti-GFP antibodies at 1:100, respectively. Sections were then incubated for $30 \mathrm{~min}$ with a goat antirabbit secondary antibody conjugated to peroxidase (Immunotech).

\section{RESULTS}

\section{Assessment of hNIS and GFP Gene Expression in RAW264.7 and $R_{\text {NIS }}$ Cells}

Flow cytometric studies demonstrated the enriched GFPpositive cell fraction $(92.5 \%)$ in $\mathrm{R}_{\mathrm{NIS}}$ cells but not in parental RAW264.7 cells (Supplemental Fig. 2). hNIS-specific immunoreactivity was also observed in $\mathrm{R}_{\mathrm{NIS}}$ cells by immunofluorescent staining (Fig. 1). In contrast, parental RAW264.7 cells were not specifically stained with antihNIS antibody. Isotype control did not show the hNISspecific immunoreactivity in $\mathrm{R}_{\mathrm{NIS}}$ cells.

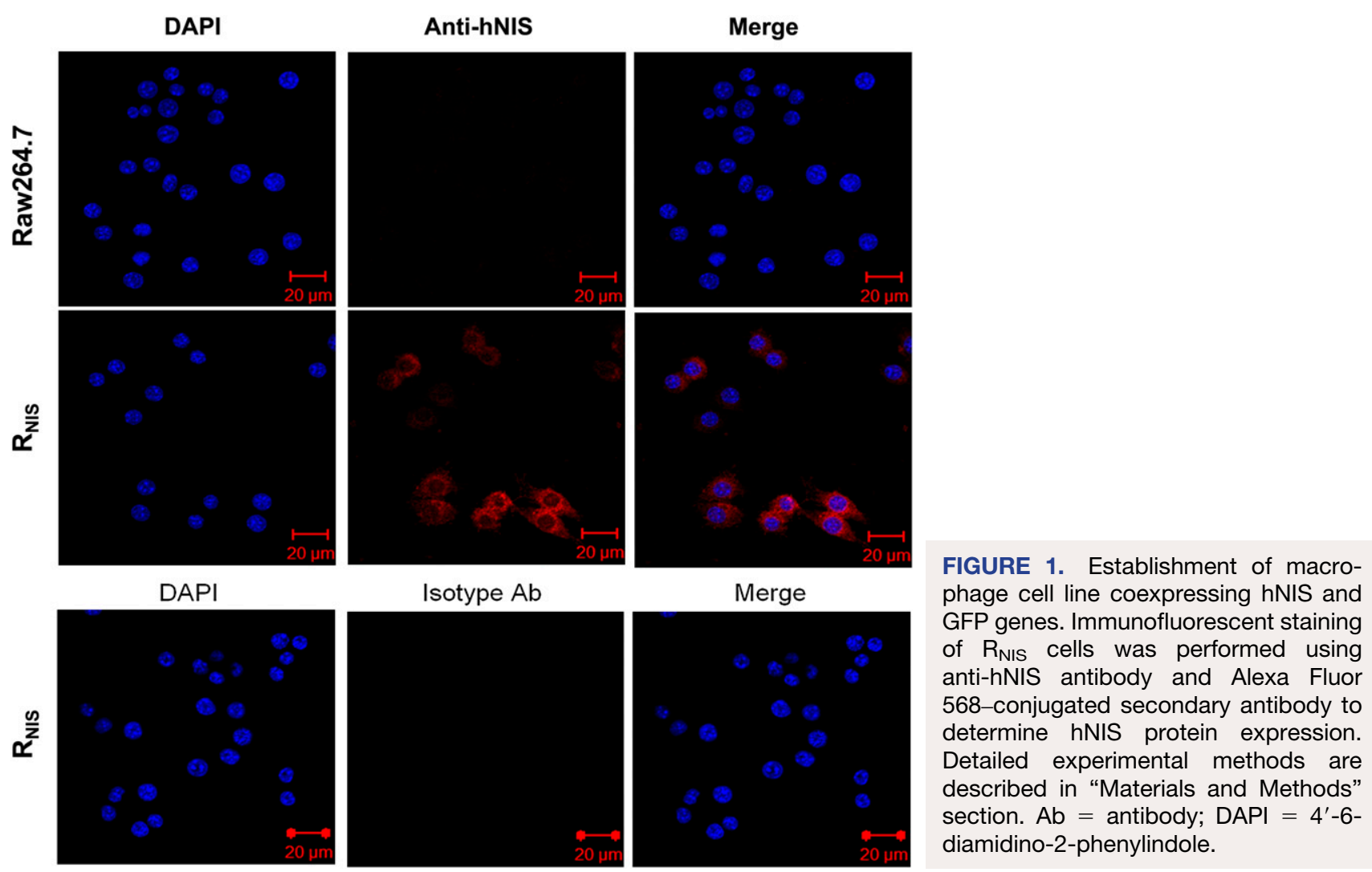


Radioiodide Uptake Assays in $\mathbf{R}_{\mathrm{NIS}}$ cells

${ }^{125}$ I uptake increased proportionally with increases in cell number for $\mathrm{R}_{\mathrm{NIS}}$ cells but not for parental RAW264.7 cells (Fig. 2A, top). Iodide uptake by $\mathrm{R}_{\mathrm{NIS}}$ cells was about 75 -fold higher than that of RAW264.7 cells. The efflux of ${ }^{125}$ I was relatively rapid and the half-time was around $5 \mathrm{~min}$ (Fig. 2A, bottom).

\section{Effects of Viral Infection on Cell Proliferation and Cytokine Production of Macrophages}

Cell proliferation assay showed that there was no significant difference in cell proliferation activity between parental RAW264.7 cells and $\mathrm{R}_{\mathrm{NIS}}$ cells (Supplemental Fig. 3). In the absence of lipopolysaccharide, the levels of proinflammatory cytokine, such as TNF- $\alpha$ and IL-12p70, were not different between parental RAW264.7 cells and $\mathrm{R}_{\mathrm{NIS}}$ cells (Fig. 3). Lipopolysaccharide treatment resulted in about 2-fold increases of proinflammatory cytokine production in both cell lines, but there was no difference in cytokine production between RAW264.7 and $\mathrm{R}_{\mathrm{NIS}}$ cells.

\section{Measurement of Phagocytic Activity}

The phagocytic activity of $\mathrm{R}_{\mathrm{NIS}}$ cells was assayed with FACS analysis of $\mathrm{R}_{\mathrm{NIS}}$ cells engulfing 7-AAD-stained E. coli, and data were compared with those of RAW 264.7 cells (Supplemental Fig. 4). The phagocytic activity assay demonstrated that the uptake of 7-AAD-stained E. coli was increased in both RAW264.7 and $\mathrm{R}_{\mathrm{NIS}}$ cells at $37^{\circ} \mathrm{C}$, but there were no significant differences between the 2 cell lines (RAW264.7 and $\mathrm{R}_{\mathrm{NIS}}$ cells, 32.1\% $\pm 0.8 \%$ and $32.74 \% \pm 0.98 \%$, respectively). As shown in Supplemental Figure 4B, phagocytic activity of the macrophages was markedly reduced with incubation at $4^{\circ} \mathrm{C}$ both in RAW264.7 and in $\mathrm{R}_{\mathrm{NIS}}$ cells but not significantly different $(12.01 \% \pm 0.3 \%$ and $11.55 \% \pm 0.10)$.

\section{$\gamma$-Camera Imaging of $\mathbf{R}_{\text {NIS }}$ Cells}

${ }^{99 m}$ Tc-pertechnetate $\gamma$-camera images of the mice acquired after inoculation of $8 \times 10^{6}$ and $2 \times 10^{7} \mathrm{R}_{\mathrm{NIS}}$ cells revealed focal tracer accumulations in the right and left thighs of mice injected with $\mathrm{R}_{\mathrm{NIS}}$ cells (Fig. 2B), and the uptake of tracer by the hNIS-expressing macrophage $\left(\mathrm{R}_{\mathrm{NIS}}\right)$ was increased proportionally with the increase in cell numbers $\left(8 \times 10^{6}\right.$ and $2 \times 10^{7} \mathrm{R}_{\mathrm{NIS}}$ cells, $2.83 \pm 1.14$-fold and $4.56 \pm 1.04$-fold, respectively).

\section{Small-Animal PET of Macrophage Migration to Inflammation Site}

As shown in Figure 4A, turpentine oil was administered intramuscularly into the right thigh of nude mice to induce the inflammation, and a focal inflammatory lesion was confirmed. After skinning the inflammation tissue, the inflamed area was more clearly observed by visual inspection (Fig. 4A).

${ }^{18}$ F-FDG PET demonstrated a donut-shaped hot spot at the inflammation site of mice administered RAW264.7 cells or $\mathrm{R}_{\mathrm{NIS}}$ cells (Fig. 4B, left). Small-animal PET with ${ }^{124}$ I also clearly demonstrated the donut-shaped focal uptake at the corresponding inflammation site of mice

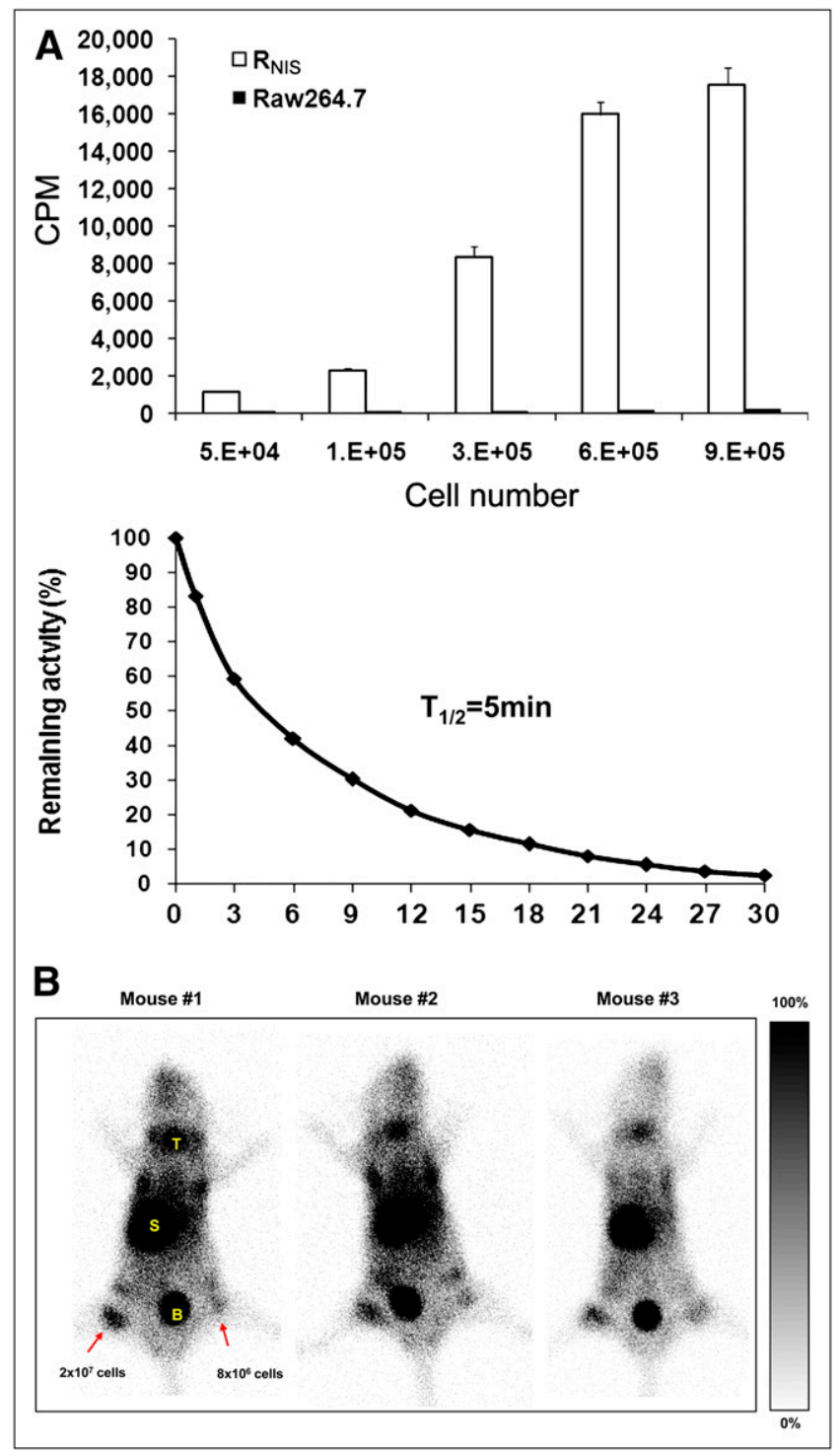

FIGURE 2. Validation of hNIS expression in vitro. (A) In vitro kinetics of ${ }^{125} \mathrm{I}$ in macrophage cells expressing hNIS gene are presented. Differences in ${ }^{125}$ uptake are according to cell numbers and ${ }^{125}$ efflux study in $\mathrm{R}_{\text {NIS }}$ cells. Half-time $\left(T_{1 / 2}\right)$ of ${ }^{125} \mathrm{I}$ efflux was about $5 \mathrm{~min}$. Detailed experimental methods are described in "Materials and Methods" section. Data are expressed as mean $\pm \mathrm{SD}$. (B) Scintigraphic imaging of nude mice implanted with $R_{\text {NIS }}$ cells. $R_{\text {NIS }}\left(8 \times 10^{6}\right.$ and $2 \times 10^{7}$ ) cells were subcutaneously inoculated in right and left thighs of nude mice, respectively. Twenty-four hours later, scintigraphic imaging was acquired after intravenous injection of $18.5 \mathrm{MBq}$ of $99 \mathrm{mTc}$-pertechnetate in nude mice. In both thighs of mice inoculated with $R_{\text {NIS }}$ cells, there was focal tracer accumulation proportional to increases in cell numbers. Physiologic iodide uptake was shown in thyroid, stomach, and urinary bladder. $\mathrm{B}=$ bladder; $\mathrm{CPM}=$ counts per minute; $\mathrm{S}=$ stomach; $\mathrm{T}=$ thyroid.

administered $\mathrm{R}_{\mathrm{NIS}}$ cells (Fig. 4B, right, bottom). The hot-spot area on ${ }^{124}$ I PET images was compatible with that on ${ }^{18} \mathrm{~F}$-FDG PET images, which corresponds to the area of focal inflammation by microscopic examination of mice 


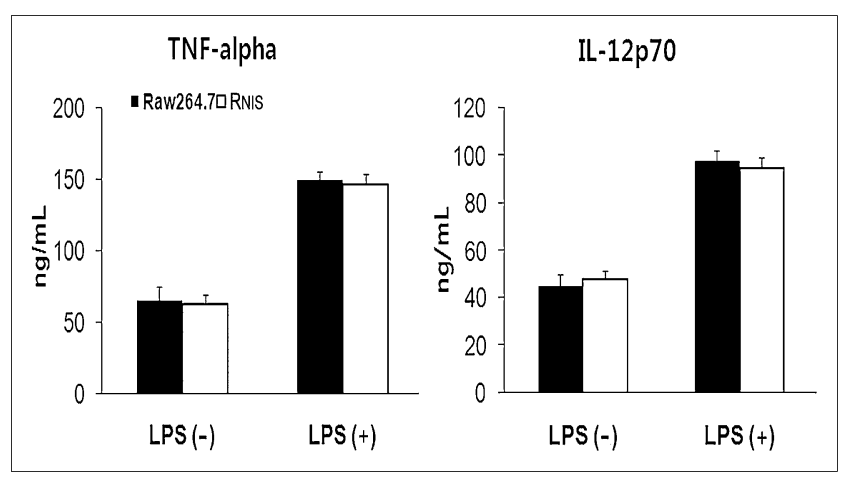

FIGURE 3. Effect of reporter gene transduction on proliferation and cytokine production (by lipopolysaccharide treatment) of macrophage cells. Cells were stimulated with lipopolysaccharide $(1 \mu \mathrm{g} / \mathrm{mL})$, and then $24 \mathrm{~h}$ later, culture supernatant was analyzed to determine amount of IL12 p70 and TNF- $\alpha$ using enzyme-linked immunosorbent assay. Data are expressed as mean \pm SD. LPS = lipopolysaccharide.

administered $\mathrm{R}_{\mathrm{NIS}}$ cells. But the ${ }^{124} \mathrm{I}$ uptake was not detected at the inflammation site of mice injected with parental RAW264.7 cells (Fig. 4B, right, upper). The percentage injected dose per gram in the region of interest drawn on the inflammation site was 2.12 times higher in mice injected with $\mathrm{R}_{\mathrm{NIS}}$ cells than in mice injected with parental RAW264.7 cells $(5.32 \pm 0.58$ vs. $2.51 \pm 0.35$, $P=0.027$; Fig. 4C).
Immunohistochemistry

Histopathologic examinations revealed extensive coagulation necrosis at the site of turpentine oil injection and viable foamy macrophages in surrounding necrotic foci of inflamed skeletal muscle administered RAW264.7 cells. As shown in Figure 5, immunohistochemical staining did not show any reactivity for GFP and hNIS at the inflammation site of the mice injected with parental RAW264.7 cells. On the other hand, at the inflammation site of mice administered $\mathrm{R}_{\mathrm{NIS}}$ cells, the cells showed diffuse and strong positive staining for GFP with cytoplasmic pattern. The macrophages accumulated in the lesion showed diffuse and strong positivity for hNIS with cytoplasmic membrane pattern.

\section{DISCUSSION}

We have successfully demonstrated the migration of macrophages to inflamed tissue in an animal model by hNIS gene transfection and ${ }^{124} \mathrm{I}$ small-animal PET.

Early attempts were made to visualize the macrophages in vivo, using radionuclides such as ${ }^{111} \mathrm{In}$ and ${ }^{125} \mathrm{I}$ (14-16). In the era of molecular imaging, optical imaging using enhanced GFP and Fluc genes $(17,18)$ and 1,1-dioctadecyl-3,3,3,3-tetramethylindotricarbocyanine iodide cell tracker dyes (19), MRI using superparamagnetic iron oxide $(20,21)$, and CT using a nanoparticulate contrast agent (22) also have been applied for macrophage trafficking.

Although nuclear medicine imaging with reporter gene technology has been widely accepted in various fields, it has not yet been successfully applied for trafficking

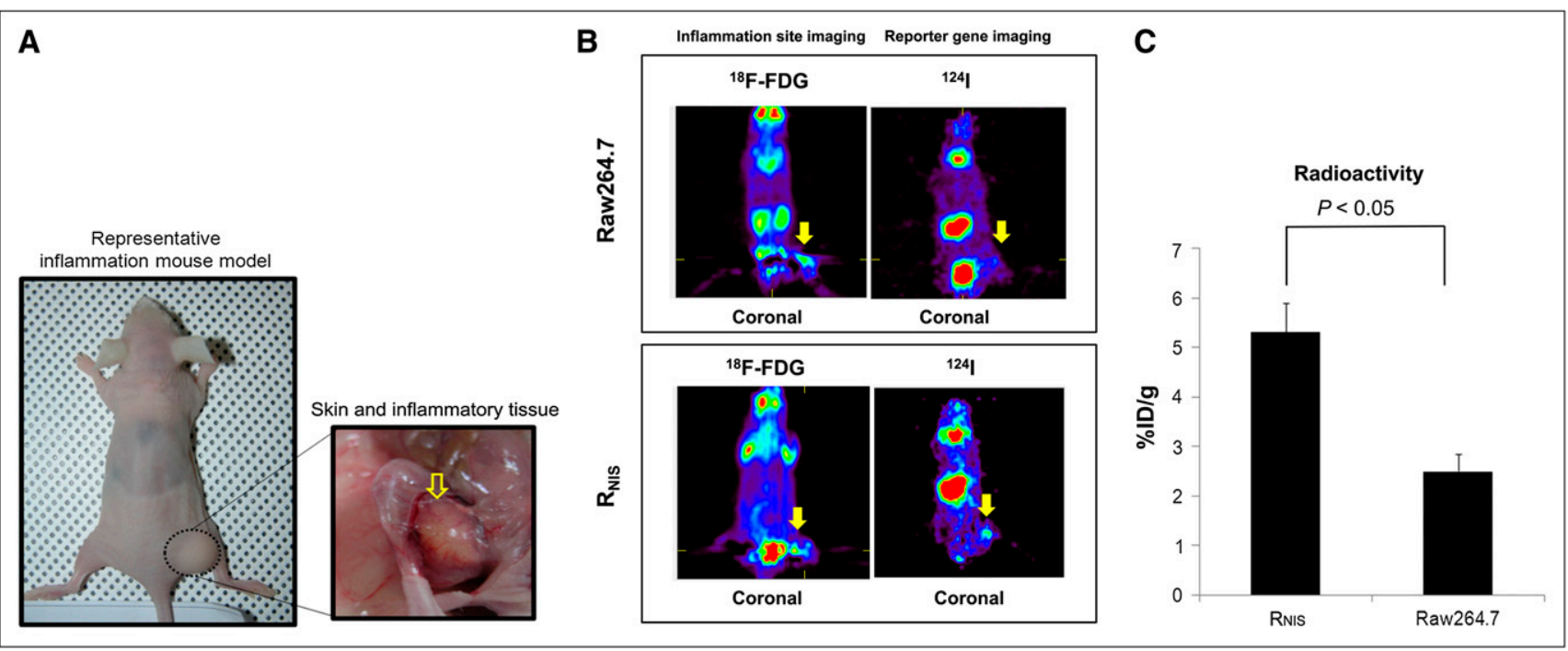

FIGURE 4. Small-animal PET in focal inflammation model. (A) Representative photograph of inflammation model induced by turpentine oil. Yellow arrows indicate stripped inflammatory tissue. (B) Small-animal PET images of each mouse group administered parental RAW264.7 or $\mathrm{R}_{\mathrm{NIS}}$ cells. (Left) ${ }^{18} \mathrm{~F}-\mathrm{FDG}$ PET scan demonstrates donut-shaped hot spot at inflammation sites of mice administered RAW264.7 and $\mathrm{R}_{\text {NIS }}$ cells. (Right) ${ }^{124}$ I PET scan also clearly demonstrates donut-shaped focal uptake at inflammation site of mice administered $R_{\text {NIS }}$ cells. Hot spot of PET images with ${ }^{124}$ I correlated strongly with that of ${ }^{18} \mathrm{~F}-\mathrm{FDG}$ PET images at inflammation site of mice administered $R_{\text {NIS }}$ cells, but tracer uptake was not observed at inflammation site of mice injected with parental RAW264.7 cells. (C) lodine accumulation was 2.17 times higher in region of interest drawn over inflammation site of mice injected with $\mathrm{R}_{\mathrm{NIS}}$ cells. \%ID/g = percentage injected dose per gram. 


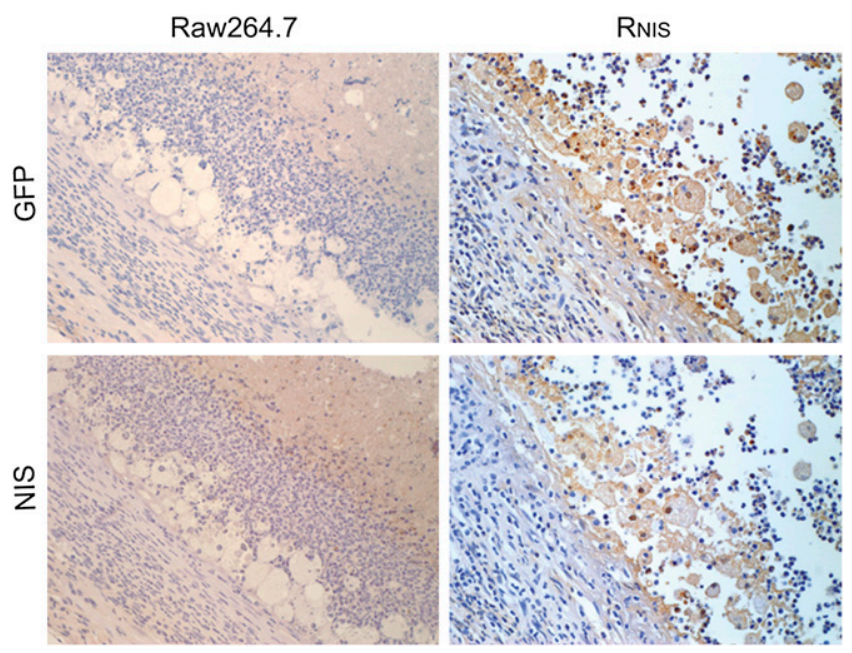

FIGURE 5. Immunohistochemical analysis of hNIS and GFP genes. In inflammation site of mice administered RAW264.7 cells, skeletal muscle showed extensive coagulation necrosis with viable foamy macrophages. By immunohistochemistry, RAW264.7 cells revealed negative reactivity for GFP and NIS $(\times 400)$. In inflammation site of mice administered $R_{\text {NIS }}$ cells, cells showed diffuse and strong positive staining for GFP with cytoplasmic patterns. Also, macrophages revealed diffuse and strong positivity for NIS with cytoplasmic membrane pattern $(\times 400)$.

macrophage migration in vivo. In a recent study, Ren et al. have successfully transfected a murine macrophage cell line RAW264.7 with a bioluminescent reporter gene and demonstrated sequential noninvasive imaging in vivo using bioluminescence (18). However, the delivery of recombinant genetic constructs into macrophages is generally known to be difficult, which limits the use of powerful molecular approaches to the studies of the macrophage biology. It is known that the widely accepted transfection methods using synthetic carriers to deliver naked plasmid DNA are inefficient in primary macrophages (23). Only a few monocyte or macrophage cell lines, such as THP-1 or RAW264.7, can be efficiently transfected by plasmid DNA. Recombinant vectors based on adenovirus and lentivirus had been used to deliver genetic constructs into macrophages much more efficiently (24). Ex vivo manipulation, genetically (e.g., using gene coding for activating cytokines such as interferon- $\alpha$ ) or by treatment with activating agents such as cytokines or lipopolysaccharide, can enhance the ability of adoptively transferred macrophages to migrate into diseased tissues and enables them to carry DNA constructs into such sites (1). Previous studies that focused on macrophage homing have found the presence of labeled macrophages at tumor or wound sites to persist for only about 6-7 d after systemic administration in cases of primary macrophages $(15,25)$. Meanwhile, if stable transduction of the gene has been achieved, reporter gene-encoding products will be continuously expressed as long as the cell is viable, even after cell division (26).
Stable transduction is obtained by ex vivo transfer using retroviral or lentiviral vectors, which result in incorporation of the reporter gene into the cell genome (9). Thus, the immortalized macrophage cell line RAW264.7 and lentiviral vectors were chosen as an integrating system to obtain stable expression of the hNIS protein in this study.

In the current study, both hNIS and GFP genes were transduced into RAW264.7 cells via the lentiviral vector system. GFP is a widely used, convenient marker for assessing in vitro and in vivo gene transduction, because its expression can be readily detected in live cells and tissues by flow cytometry or fluorescent microscopy. In addition, hNIS has the benefit of being able to image with easily available radiotracers such as ${ }^{99 \mathrm{~m}} \mathrm{Tc}$-pertechnate and radioiodine and nuclear imaging instruments, thus potentiating its clinical application. Coexpression of hNIS and GFP genes was confirmed by flow cytometric study and immunofluorescent staining. The blue fluorescent nuclei and red fluorescent membranes were clearly demonstrated with the confocal microscopic analysis using 4'-6-diamidino-2-phenylindole and anti-hNIS stain. Also, significant increases in iodine uptake in $\mathrm{R}_{\mathrm{NIS}}$ cells, compared with parent cells, were observed, and the degree of uptake was proportional to the cell numbers. Functional expression of the hNIS gene in $\mathrm{R}_{\mathrm{NIS}}$ cells was also verified in vivo by nuclear imaging with ${ }^{99 \mathrm{~m}} \mathrm{Tc}$-pertechnetate of mice with subcutaneously implanted $\mathrm{R}_{\mathrm{NIS}}$ cells. The cell proliferation and proinflammatory cytokine production activity were not altered by the transduction of the dual reporter gene system, suggesting that the cell growth and cytokine production needed in the inflammatory process are preserved as in the parent cells. In addition, the phagocytic activity, which is the most important function of the macrophage, was not affected, when assessed with incorporated 7-AAD measurement, using a method for phagocytic activity measurement reported previously (10-13).

For in vivo imaging to assess macrophage migration, soft-tissue inflammation was induced by intramuscular inoculation of turpentine oil into the right thigh of the nude mice because this local inflammation model had been validated in previous studies $(27,28)$. $\mathrm{R}_{\mathrm{NIS}}$ cells were injected at $7 \mathrm{~d}$ after the induction of local inflammation, which minimizes the acute inflammatory reaction and reflects mainly the effect of chronic inflammation characterized by mononuclear cell infiltration. Imaging of the inflammation animal model was performed on the seventh day after $\mathrm{R}_{\mathrm{NIS}}$ cell injection; day 7 was chosen for imaging in this study on the basis of the results of an optical imaging study that used reporter RAW264.7 stable cell lines, in which the authors reported that significantly higher bioluminescent signals were emitted from day 6 onward (18). Injected macrophages were seen to be distributed in the lung for the first day, then circulated and migrated to the liver, spleen, and finally to the site of inflamed tissue for 
the next few days. Likewise, $7 \mathrm{~d}$ after macrophage injection, ${ }^{124}$ I PET demonstrated a donut-shaped pattern of tracer accumulations along the rim of the inflammatory tissue in mice injected with $\mathrm{R}_{\mathrm{NIS}}$ cells. Similarly, intense ${ }^{18} \mathrm{~F}-\mathrm{FDG}$ uptake reflected the active inflammation that was observed along the rim of the inflammatory tissue, with a central photon-deficient area corresponding to coagulation necrosis along with core turpentine oil. On the other hand, no ${ }^{124}$ I uptake was shown at the inflammation site of the mice injected with parental RAW264.7 cells. The findings of histologic examination confirmed the migration of $\mathrm{R}_{\mathrm{NIS}}$ cells to the inflammation site. The macrophages showing strong positive staining for GFP and hNIS were recruited around the inflammation site in the skeletal muscle of the mice.

After we successfully localized the NIS-transfected macrophages in inflammation lesions with nuclear imaging, animals were sacrificed for further verification of the localized macrophage cells with immunohistochemical staining. Thus, an image of the long-term trafficking of microphage migration from early ( 2 and $4 \mathrm{~d}$ after cell injection) to late times was not provided in this study. To extend the macrophage migration study, visualization of the macrophage migration using a dual-reporter gene, such as firefly luciferase and hNIS gene, would be helpful. We presume that dual-reporter genes expressing macrophages may show the macrophage migration and proliferation in superficial or deep tissue from early to late times.

This could be the first study to implement nuclear reporter gene imaging using hNIS genes to monitor macrophage migration toward inflamed tissue. The current study offers the possibility of developing immune cell trafficking imaging using reporter genes. Immune cells such as macrophages and dendritic cells may be used in extensive research on inflammatory processes, immune systems, or tumor pathology through nuclear molecular imaging.

\section{CONCLUSION}

Immortalized macrophage RAW264.7 cells were successfully transduced with hNIS genes for trafficking. Macrophage migration to the local inflammation site was demonstrated by in vivo imaging using ${ }^{124} \mathrm{I}$ and also by histopathologic examination. These data support the feasibility of reporter gene imaging using hNIS to monitor macrophage migration toward inflamed tissue. Macrophages expressing hNIS may provide a new strategy to investigate the inflammatory response in preclinical models.

\section{ACKNOWLEDGMENTS}

This work was supported by the Basic Atomic Energy Research Institute (BAERI, 2010-0017515) and BK21 (2009) and the Korean Ministry of Education, Science and Technology (The Regional Core Research Program/ Medical Convergence Technology Development Consortium for Anti-aging and Well-being).

\section{REFERENCES}

1. Burke B, Sumner S, Maitland N, Lewis CE. Macrophages in gene therapy: cellular delivery vehicles and in vivo targets. J Leukoc Biol. 2002;72:417-428.

2. Feldmann M, Steinman L. Design of effective immunotherapy for human autoimmunity. Nature. 2005;435:612-619.

3. Orlic D, Kajstura J, Chimenti S, et al. Bone marrow cells regenerate infarcted myocardium. Nature. 2001;410:701-705.

4. Ross JAAM. The biology of the macrophage. In: Burke B, Lewis CE, eds. The Macrophage. 2nd ed. Oxford, U.K.: Oxford University Press; 2002.

5. Boerman OC, Rennen H, Oyen WJ, Corstens FH. Radiopharmaceuticals to image infection and inflammation. Semin Nucl Med. 2001;31:286-295.

6. Mulder WJ, Strijkers GJ, van Tilborg GA, Griffioen AW, Nicolay K. Lipid-based nanoparticles for contrast-enhanced MRI and molecular imaging. NMR Biomed. 2006;19:142-164

7. Gambhir SS. Molecular imaging of cancer with positron emission tomography. Nat Rev Cancer. 2002;2:683-693.

8. Serganova I, Blasberg R. Reporter gene imaging: potential impact on therapy. Nucl Med Biol. 2005;32:763-780.

9. Bengel FM, Schachinger V, Dimmeler S. Cell-based therapies and imaging in cardiology. Eur J Nucl Med Mol Imaging. 2005;32(suppl 2):S404-S416.

10. Gaforio JJ, Serrano MJ, Algarra I, Ortega E, Alvarez de Cienfuegos G. Phagocytosis of apoptotic cells assessed by flow cytometry using 7-aminoactinomycin D. Cytometry. 2002;49:8-11.

11. Grunwald U, Fan X, Jack RS, et al. Monocytes can phagocytose gram-negative bacteria by a CD14-dependent mechanism. J Immunol. 1996;157:4119-4125.

12. Brulez HF, ter Wee PM, Snijders SV, Donker AJ, Verbrugh HA. Mononuclear leucocyte function tests in the assessment of the biocompatibility of peritoneal dialysis fluids. J Clin Pathol. 1999;52:901-909.

13. Won DI, Heo WB, Suh JS. Age-related decrease and a simple flow cytometric assay of neutrophil function. Korean J Lab Med. 2005;25:273-279.

14. Abreo K, Lieberman LM, Moorthy AV. Distribution studies of ${ }^{111}$ In-oxinelabeled peritoneal mononuclear cells in tumor-bearing rats. Int $\mathrm{J} \mathrm{Nucl} \mathrm{Med}$ Biol. 1985;12:53-55.

15. Audran R, Collet B, Moisan A, Toujas L. Fate of mouse macrophages radiolabelled with PKH-95 and injected intravenously. Nucl Med Biol. 1995;22:817-821.

16. Faradji A, Bohbot A, Frost H, et al. Phase I study of liposomal MTP-PEactivated autologous monocytes administered intraperitoneally to patients with peritoneal carcinomatosis. J Clin Oncol. 1991;9:1251-1260.

17. Pastorino S, Massazza S, Cilli M, Varesio L, Bosco MC. Generation of high-titer retroviral vector-producing macrophages as vehicles for in vivo gene transfer. Gene Ther. 2001;8:431-441.

18. Ren PG, Lee SW, Biswal S, Goodman SB. Systemic trafficking of macrophages induced by bone cement particles in nude mice. Biomaterials. 2008;29:4760-4765.

19. Eisenblatter M, Ehrchen J, Varga G, et al. In vivo optical imaging of cellular inflammatory response in granuloma formation using fluorescence-labeled macrophages. J Nucl Med. 2009;50:1676-1682.

20. Kaim AH, Jundt G, Wischer T, et al. Functional-morphologic MR imaging with ultrasmall superparamagnetic particles of iron oxide in acute and chronic softtissue infection: study in rats. Radiology. 2003;227:169-174.

21. Ruehm SG, Corot C, Vogt P, Kolb S, Debatin JF. Magnetic resonance imaging of atherosclerotic plaque with ultrasmall superparamagnetic particles of iron oxide in hyperlipidemic rabbits. Circulation. 2001;103:415-422.

22. Hyafil F, Cornily JC, Feig JE, et al. Noninvasive detection of macrophages using a nanoparticulate contrast agent for computed tomography. Nat Med. 2007;13:636-641.

23. Heider H, Verca SB, Rusconi S, Asmis R. Comparison of lipid-mediated and adenoviral gene transfer in human monocyte-derived macrophages and COS-7 cells. Biotechniques. 2000;28:260-265, 268-270.

24. Pan H, Mostoslavsky G, Eruslanov E, Kotton DN, Kramnik I. Dual-promoter lentiviral system allows inducible expression of noxious proteins in macrophages. J Immunol Methods. 2008;329:31-44.

25. Chokri M, Lallot C, Ebert M, Poindron P, Batholeyns J. Biodistribution of indium-labelled macrophages in mice bearing solid tumors. Int $J$ Immunotherapy. 1990;6:79-84.

26. Higuchi T, Anton M, Dumler K, et al. Combined reporter gene PET and iron oxide MRI for monitoring survival and localization of transplanted cells in the rat heart. J Nucl Med. 2009;50:1088-1094.

27. Tron K, Novosyadlyy R, Dudas J, Samoylenko A, Kietzmann T, Ramadori G. Upregulation of heme oxygenase-1 gene by turpentine oil-induced localized inflammation: involvement of interleukin-6. Lab Invest. 2005;85:376-387.

28. Yamada S, Kubota K, Kubota R, Ido T, Tamahashi N. High accumulation of fluorine-18-fluorodeoxyglucose in turpentine-induced inflammatory tissue. $J$ Nucl Med. 1995;36:1301-1306. 